\title{
Three-Dimensional Radiofrequency Tissue Tightening: A Proposed Mechanism and Applications for Body Contouring
}

\author{
Malcolm Paul • G. Blugerman • M. Kreindel • \\ R. S. Mulholland
}

Received: 20 April 2010/ Accepted: 6 July 2010/Published online: 11 September 2010

(C) The Author(s) 2010. This article is published with open access at Springerlink.com

\begin{abstract}
The use of radiofrequency energy to produce collagen matrix contraction is presented. Controlling the depth of energy delivery, the power applied, the target skin temperature, and the duration of application of energy at various soft tissue levels produces soft tissue contraction, which is measurable. This technology allows precise soft tissue modeling at multiple levels to enhance the result achieved over traditional suction-assisted lipectomy as well as other forms of energy such as ultrasonic and lasergenerated lipolysis.
\end{abstract}

Keywords Body contouring - Liposuction ·

Radiofrequency energy · Soft tissue contraction

\section{Introduction}

Radiofrequency (RF) thermal-induced contraction of collagen is well known in medicine and is used in ophthalmology, orthopedic applications, and treatment of varicose veins. Each type of collagen has an optimal contraction temperature that does not cause thermal destruction of connective tissue but induces a restructuring effect in

\author{
M. Paul ( $\square)$ \\ Department of Surgery, Aesthetic and Plastic Surgery Institute, \\ University of California, Irvine, CA 92697, USA \\ e-mail: mpaulmd@hotmail.com \\ G. Blugerman \\ Buenos Aires, Argentina \\ M. Kreindel \\ Invasix Corp, Toronto, ON, Canada \\ R. S. Mulholland \\ SpaMedica, Toronto, ON, Canada
}

collagen fibers. The reported range of temperatures causing collagen shrinkage varies from 60 to $80^{\circ} \mathrm{C}$ [1-7]. At this temperature tissue contraction occurs immediately after tissue reaches the threshold temperature. The shrinkage of tissue is dramatic and can reach tens of percent of the heated tissue volume. This type of contraction is well studied in cornea [1], joints [2], cartilage [4, 7], and vascular tissue [5] but its application for skin, subdermal tissue, and subcutaneous tissue tightening has not been studied.

Noninvasive RF and lasers have been used for skintightening effects since the mid-1990s [6, 8-12]. Because of superficial thermal safety concerns, the skin surface temperature is maintained below $45^{\circ} \mathrm{C}$. To increase the temperature in the deep dermis the skin is heated with RF or laser energy penetrating into the tissues deeper than $1.5 \mathrm{~mm}$, with simultaneous skin surface cooling. This sophisticated method of transepidermal, noninvasive RF thermal delivery provides a variable and controversial tightening effect, which is not usually apparent, if at all, until dermal remodeling occurs a few months after the treatment. Noninvasive tissue tightening treatments have an inherent safety limitation because energy is delivered through the skin surface and the threshold epidermal burn temperature is significantly lower than the optimal temperature for the collagen contraction. Studies indicate that deeper penetrating energy provides better skin contraction and RF energy, by penetrating deeper than laser radiation, is a superior method, not only for treatment of facial rhytides and laxity, but also for body tightening [6, 9, 12]. It is the physical and biological characteristics of RF that explain its superior three-dimensional mechanism of skin tightening.

Recently, the use of thermal-induced tissue tightening was expanded to minimally invasive treatments [13-16]. 
Using laser-assisted liposuction (LAL) or radiofrequencyassisted liposuction (RFAL), physicians have attempted to achieve reduction of subcutaneous tissue with simultaneous tissue contraction [13, 16]. DiBernardo [13] reported 17\% skin surface shrinkage measured at 3 months follow-up after LAL treatment. RFAL technology provides much higher power and more efficient energy transfer than laser energy systems and thus allows the treatment of larger volumes of subcutaneous tissue with optimal thermal profiles, facilitating the significant tightening of the tissue. Paul and Mulholland [16] introduced a RFAL and soft tissue contraction technology that showed tremendous promise for thermal contouring. Invasive thermal treatments are superior because the RF conduit (RFAL emitting cannula) targets the whole volume of treated tissue with critical thermal energy, not only the superficial subdermal layer, and the invasive RF treatments can heat deep adipose and subcutaneous tissue to much higher temperatures without compromising skin safety.

When considering skin contraction we have to differentiate two-dimensional horizontal $x$-axis tightening of the skin surface from three-dimensional $x-y-z$ tissue tightening of the subcutaneous tissue, where the skin is also more firmly connected and adjacent to the deeper anatomical structures. If two-dimensional contraction is a function of collagen structure changes in the dermis, the three-dimensional tissue-tightening changes involve contraction of different types of collagenous tissue. We can separate the following types of collagen tissue in the subcutaneous space:

- Dermis: papillary and reticular

- Fascia: relatively thick layer of connective tissue located between muscles and skin

- Septal connective tissue: thin layers of connective tissue separating lobules of fat and connecting dermis with fascia

- Reticular fibers: framework of single collagen fibers encasing fat cells

One of the main objectives of this study was to evaluate the possibility of immediate thermal-induced subcutaneous tissue contraction and to estimate the thermal threshold of the effect. In this study we compare the threshold temperature and contraction level of different types of ex vivo collagenous tissue samples and the clinical results based on RFAL results for body contouring.

\section{Materials and Methods}

\section{Ex Vivo Experiment Setup}

An ex-vivo study was conducted to measure subcutaneous collagenous tissue contraction with simultaneous monitoring of local tissue temperature to determine the threshold temperature of the collagen shrinkage. Three types of collagenous tissue were studied for thermal-induced contraction: (1) adipose tissue with septal and reticular connective tissue, (2) dermis, and (3) fascia.

Samples of ex vivo human tissue were taken from an abdominoplasty surgery and were tested within $10 \mathrm{~min}$ of excision. Immediate thermal testing was performed to minimize changes in tissue related to long storage and temperature variation or change of liquid content, including blood and lymphatic content. The tissue samples were placed between the two BodyTite ${ }^{\mathrm{TM}}$ (Invasix Ltd., Israel) $\mathrm{RF}$ electrodes, where the small-area, internal RF-active electrodes (cannula) were placed in contact with the studied tissue and the other large-area electrode was applied to the opposite side, or epidermal side, of the sample. Large samples of subcutaneous tissue were used, allowing observation of any contraction behavior in the tissue's native environment in connection with its entire matrix structure. Two marks were placed $1 \mathrm{~cm}$ from the active internal electrode to visualize tissue displacement. The experiment design setup is shown in Fig. 1.

$\mathrm{RF}$ energy was delivered by the BodyTite device. The delivered power was $70 \mathrm{~W}$ at $1 \mathrm{MHz}$, and energy was delivered until evaporation of water from the adipocytes was observed. Video and thermal cameras (FLIR A-320) were used to monitor tissue displacement and temperature change during the treatment. The start of tissue displacement was correlated with tissue temperature to determine the contraction thermal threshold. Each experiment was repeated three times for each type of tissue to sample tissue averages and avoid measurements of random events.

In Vivo Evaluation with Radiofrequency-Assisted Liposuction (RFAL)

Twenty-four consecutive patients, 22 female and 2 male, underwent RFAL to the abdomen and hips. The average age was 39.7 years (range $=19-52$ years). The average preoperative weight was $71 \mathrm{~kg}$. The selected patients were typical patients indicated for a liposuction procedure. All patients were healthy anesthetic risks and active with no significant medical diseases. Fifteen of 24 patients had a normal BMI $(<25)$, while 9/24 patients were moderately overweight $(\mathrm{BMI}=25-30)$ and 3 patients were obese $(30<$ BMI $<32)$.

RFAL was performed using the BodyTite device. The BodyTite device deploys a handpiece to deliver radiofrequency energy to the adipose tissue and skin. The internal cannula is coated with dielectric material and has a conductive tip that emits RF energy into the adipose tissue toward the skin surface. RF energy flows between the tip of the internal cannula and external electrode creating a 
Fig. 1 Ex vivo experimental setup

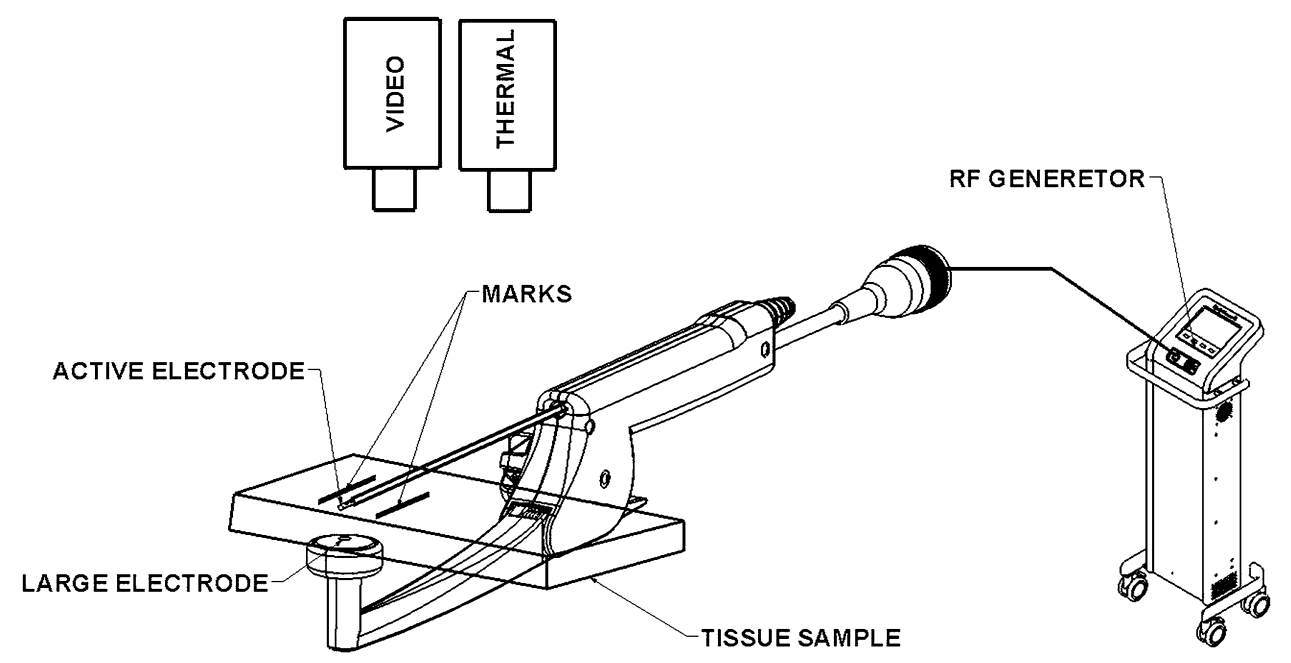

Fig. 2 Schematic drawing of $\mathrm{RF}$ handpiece inserted into the body

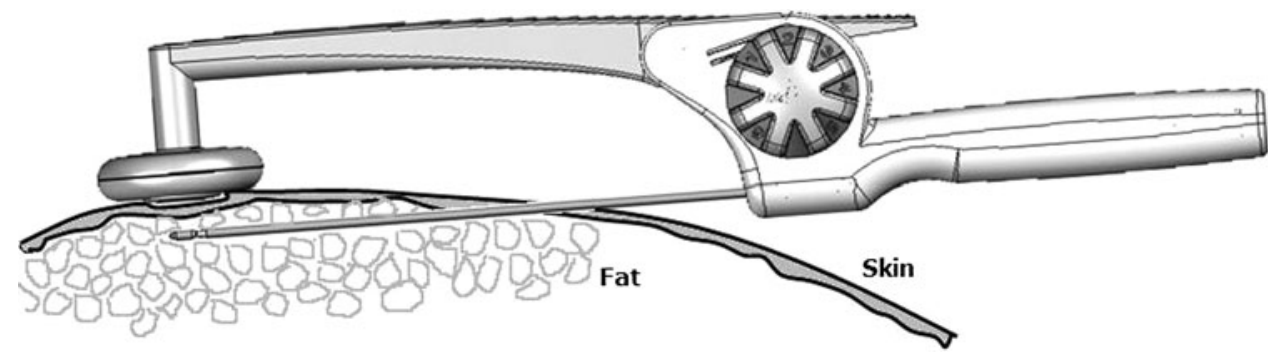

localized, confined thermal effect between them. The internal cannula is inserted into the pretumesced fat to be contoured and is moved gently back and forth at various predetermined and controlled depths for uniform heating of the treated volume. There is also an external electrode that moves along the surface of the skin in tandem vertical alignment with the tip of the internal cannula (Fig. 2). The subcutaneous tissue and skin between the electrodes experience a significant thermal effect which is maximal near the tip of the internal cannula and decreases in intensity toward the skin electrode The operator controls the depth of the internal cannula within a predetermined range of 5-50 $\mathrm{mm}$ and moves the handpiece back and forth through the desired fat volume to be contoured. The RF energy coagulates the adipose, connective, and vascular tissues in the vicinity of the internal cannula tip and gently heats the dermis below the external electrode. The internal electrode also serves as an asynchronous internal suction cannula, aspirating the coagulated adipose, vascular, and fibrous tissues.

The RF power, in the range of 40-70 W, was used for uniform heating throughout a thick subcutaneous flap. The average total energy of about $72 \mathrm{~kJ}$ was delivered to the abdominal area. The temperature around the tip of the cannula reached $70-80^{\circ} \mathrm{C}$. This internal temperature was observed using thermography on tissue cross section for preabdominoplasty patients treated with RFAL when the

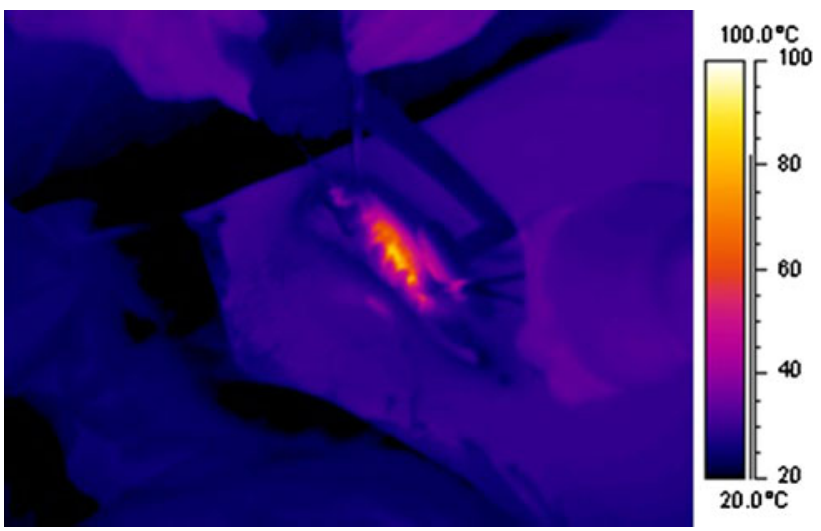

Fig. 3 Temperature profile inside adipose tissue during the RFAL treatment

skin surface temperature reached $38-42^{\circ} \mathrm{C}$ (see Fig. 3, cross section of lower abdominal tissue showing the thermal image of the skin surface and tissue incision allowing visualization of the thermal profile of the internal subcutaneous temperature). The target skin temperature was monitored and controlled with a thermal sensor built into the external electrode. The sensor provides continuous realtime epidermal temperature monitoring and feedback loop control of RF power. The system was set to a target temperature of $38-42^{\circ} \mathrm{C}$, which was maintained for $1-3 \mathrm{~min}$. The strong and sustained tissue heating during the 
Fig. 4 Before and after RFAL and intraoperative two-point linear contraction registration points from pubic RFAL incision point to the lower pole of the umbilicus
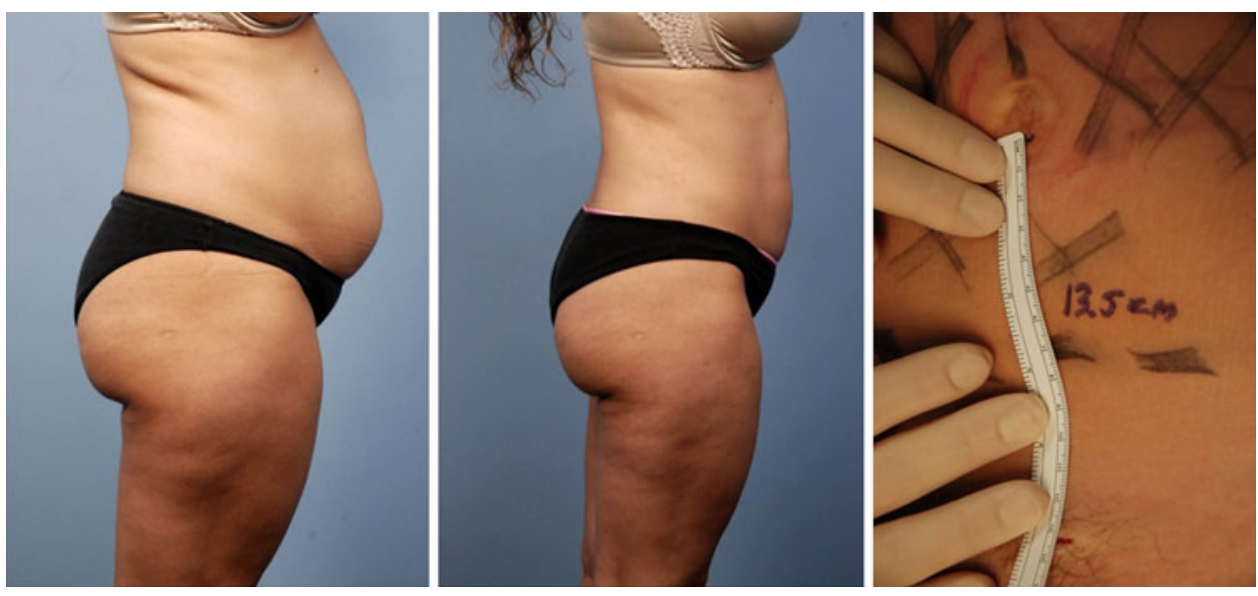

procedure results in thermal stimulation of the subdermal layer, the entire matrix of adipose tissue, and the vertical and oblique fibrous septa, eliciting a powerful threedimensional retraction and contraction of the entire soft tissue envelope.

The distance between the internal and external electrodes was controlled with an eccentric spring-loaded mechanism that keeps the external electrode on the surface of the skin at all times. The device also controls vaporization and prevents carbonization around the tip of the cannula. When evaporation around the internal cannula occurs, the tissue impedance rises and exceeds the online monitored high impedance and the device shuts off the RF energy.

All patients had their treatment area infiltrated with tumescent anesthesia prior to the RFAL procedure. Tumescent anesthesia is critical in the technique as the RF current travels through tissue most efficiently in a salinated environment.

The objective of this in vivo portion of the study was to optimize treatment parameters and correlate treatment soft tissue contraction results with procedure and patient variables, including amount of deposed RF energy, body mass index (BMI) of the patients, and amount of aspirated fat.

A zone measuring as large as $15 \times 10 \mathrm{~cm}\left(150 \mathrm{~cm}^{2}\right)$ may be heated to critical target temperature within 3-8 min depending on the thickness of the treated fat layer and then uniform volumetric heating can be safely performed to reach uniform temperature distribution over the entire treated volume.

All patients from the study were followed up at 6, 12, and 24 weeks. To measure linear contraction, the distance between two fixed points was measured preoperatively and then at the 24-week postoperative visit. Distances between incision ports and natural "fixed" anatomical registration points, such as moles or the umbilicus, were measured before the treatment, after the treatment, and at 3- and 6-month follow-up visits. The linear contraction was measured as relative change of distance between two points over the curved surface of the body. Distances were measured using a flexible ruler applied over the skin surface. For the abdominal area, at least three measurements were taken between three different points and average linear contraction was calculated (Fig. 4).

Pre- and postoperative photography, weights, and circumferential reduction data were obtained on all patients. One RFAL study patient had a biopsy of the thermally treated skin 12 months after the procedure during which epidermal skin temperatures of $40^{\circ} \mathrm{C}$ had been attained and there was an area contraction of $43 \%$ at 6 months.

\section{Results and Discussion}

\section{Ex Vivo Tissue Contraction Experiments}

The adipose tissue with septal and reticular collagen behavior is shown in Fig. 5. The experiments showed that the marker movement (contraction) started within $2 \mathrm{~s}$ after the start of RF energy delivery. Tissue contraction was not symmetrical as the displacement from one side was $8 \mathrm{~mm}$ and from the other side the average displacement was $3 \mathrm{~mm}$. Adipose fibrous septal tissue coagulation and vaporization started to be observed at $13 \mathrm{~s}$ after the initiation of RF energy. Nonsymmetrical behavior can be explained by the nonuniform structure of connective tissue and the nonsymmetrical geometry of the studied tissue sample. The average marker migration and tissue contraction for the three experiments with adipose tissue was $6.5 \mathrm{~mm}$.

Figure 6 shows thermal images of the same sample taken before the treatment, at the beginning of tissue displacement, and at the end of the treatment showing the rise in thermal profile with time and onset of contraction. For fascial tissue, contraction started when the maximal 


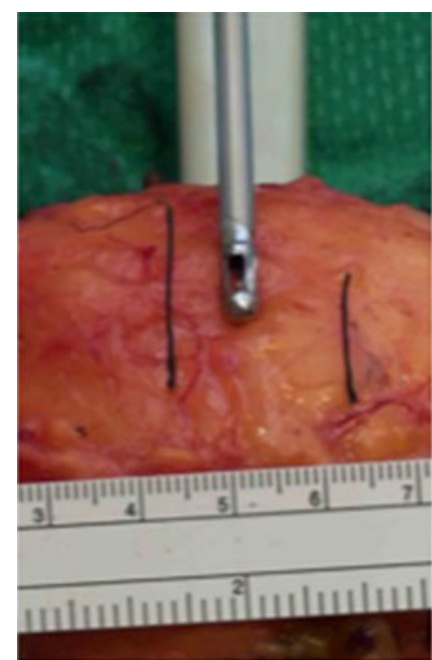

Before

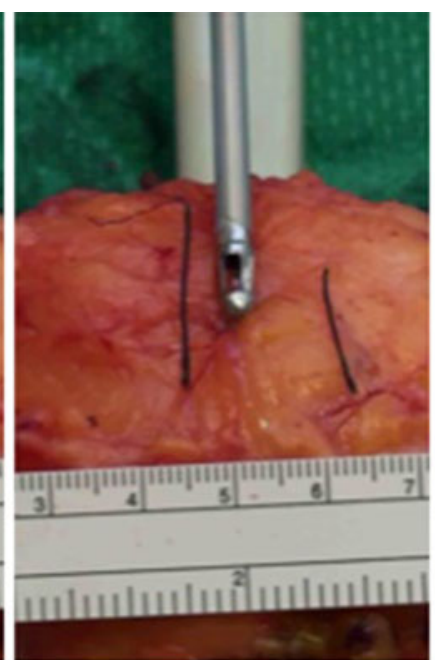

$4 \mathrm{sec}$

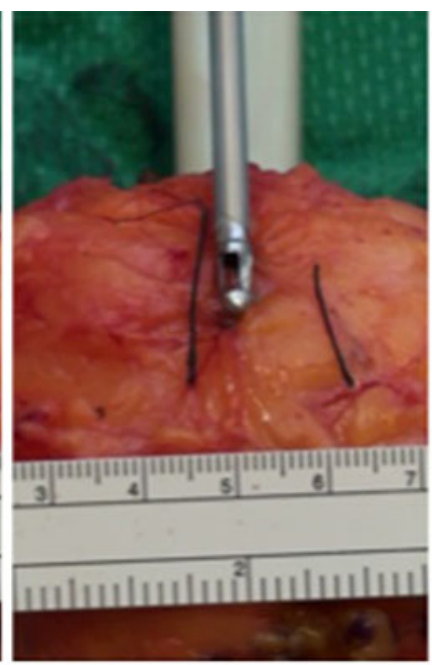

$6 \mathrm{sec}$

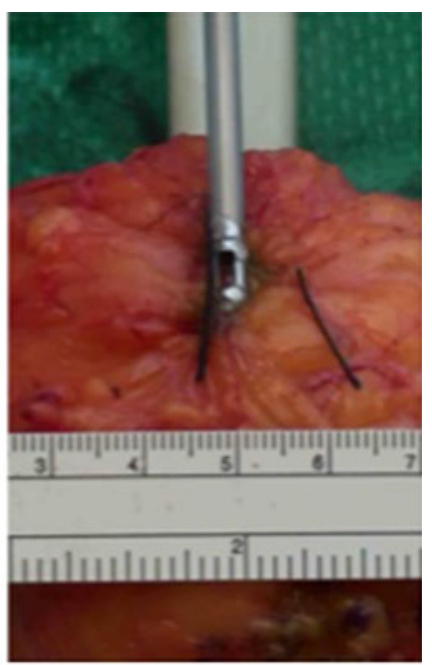

$13 \mathrm{sec}$

Fig. 5 Adipose-septal tissue behavior during RF energy delivery at different time points

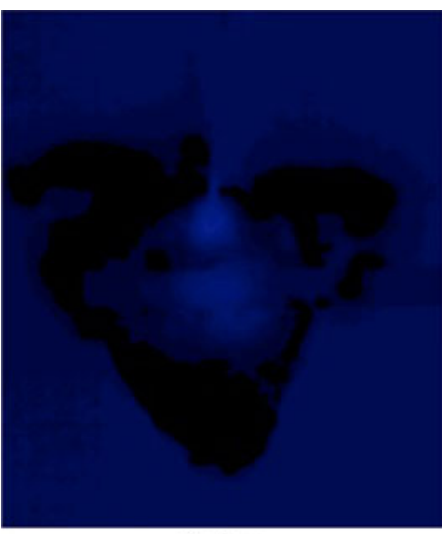

Before

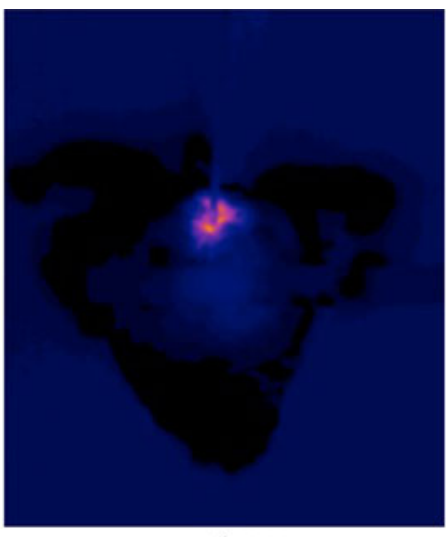

$2 \mathrm{sec}$
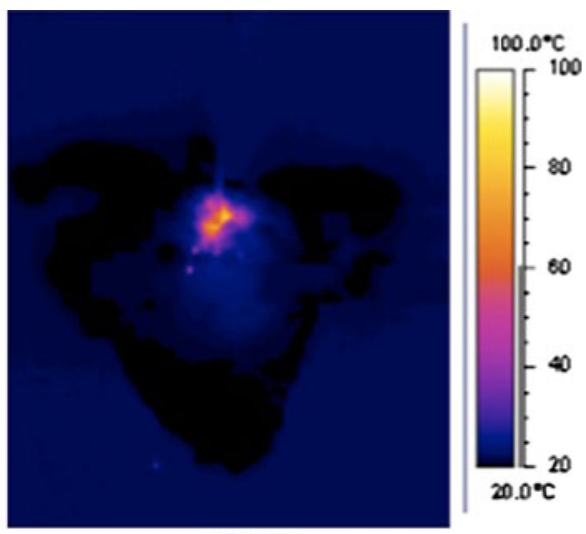

$13 \mathrm{sec}$

Fig. 6 Adipose-fibrous septal tissue thermal behavior during RF energy delivery at different time points

adipose tissue temperature near the active internal electrode reached $69.4^{\circ} \mathrm{C}$. Adipose fibrous septal tissue coagulation and vaporization started when tissue temperature reached $90-100^{\circ} \mathrm{C}$ and is most probably associated with boiling of adipocyte water content.

Fascia contraction is demonstrated in Fig. 7. The displacement of the markers and tissue contraction in fascia were significantly less than in adipose tissue. The average movement was $2.75 \mathrm{~mm}$ or approximately 2.5 times less than the mark migration and tissue contraction observed in adipose tissue. The marker migration and medial contraction started after $3.5 \mathrm{~s}$ and maximal temperature near the active electrode at this moment was $61.5^{\circ} \mathrm{C}$.

Skin behavior is presented in Fig. 8. The migration of markers and medial displacement and tissue contraction on the skin were similar to the fascia. The average movement was $2.0 \mathrm{~mm}$ or approximately 3 times less than the marker migration and contraction observed in adipose tissue. The medial marker movement started after $2.5 \mathrm{~s}$ and the maximal temperature near the active electrode during this contraction was $81.9^{\circ} \mathrm{C}$.

Table 1 summarizes the results on subcutaneous tissue contraction. From the results one can see that the strongest contraction response was observed in adipose tissue containing septal connective tissue and reticular collagen fibers encasing fat cells. The contraction temperature threshold was the highest for dermis. It is clear that the immediate contraction of dermal collagen is not possible to achieve without a skin burn, which happened when the epidermal temperature exceeded $45^{\circ} \mathrm{C}$ [13]. Fascia and septa can be heated to these high, optimal contraction temperatures, but it can be done only in a minimally invasive transcutaneous manner that deposits the thermal RF energy directly into the adipose tissue 


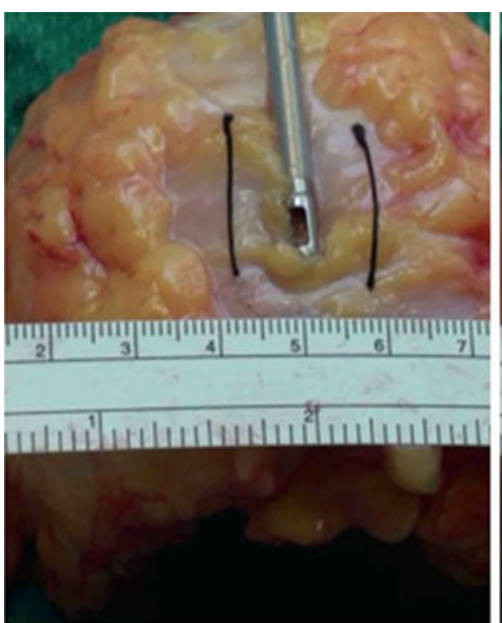

Before

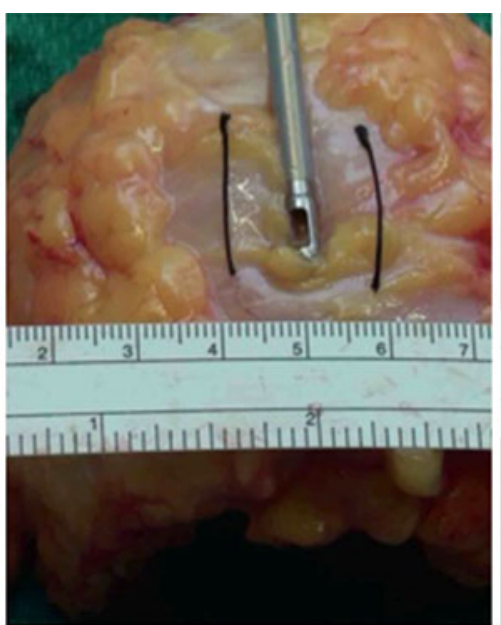

$1 \mathrm{sec}$

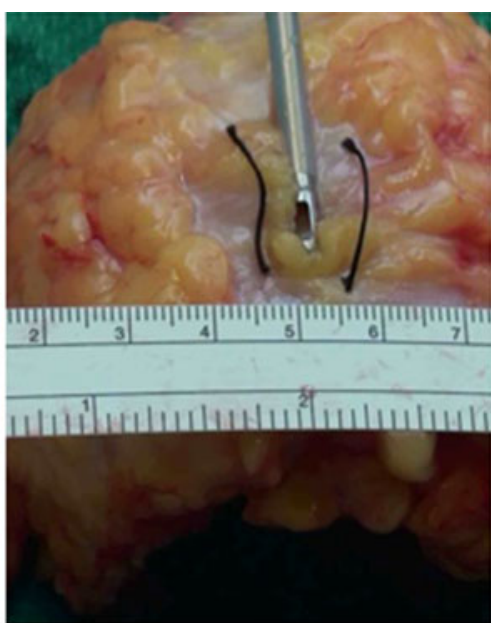

$15 \mathrm{sec}$

Fig. 7 Fascia contraction behavior during RF energy delivery at different time points

Fig. 8 Skin contraction behavior during RF energy delivery at different time points

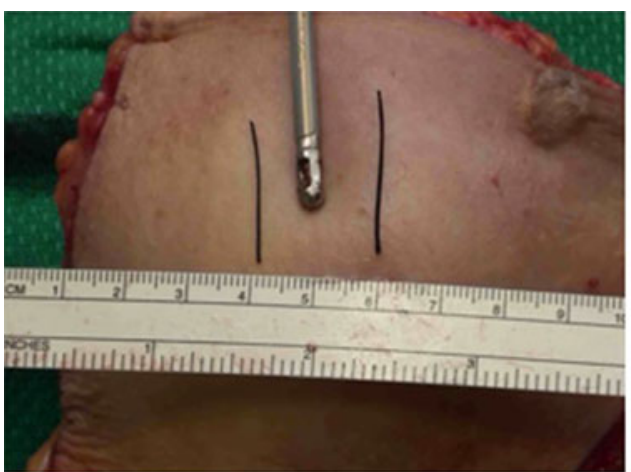

Before

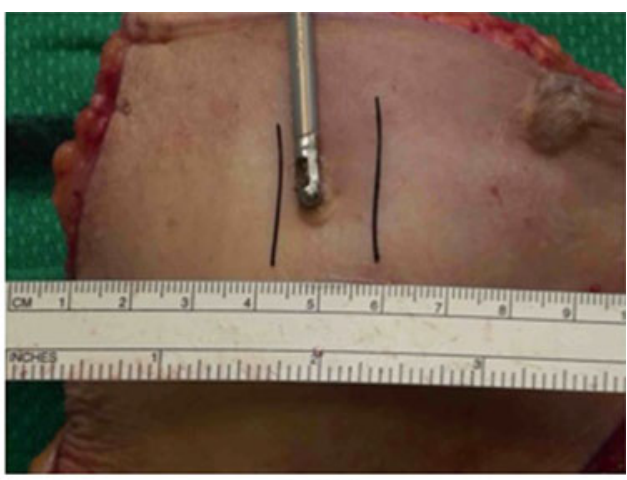

$5 \mathrm{sec}$ and subdermal space, thus avoiding heating the epidermal surfaces.

The contraction temperatures of collagen in our ex vivo study were in the same range reported for other collagenous tissues. We observed tissue contraction in the area with a diameter of $2 \mathrm{~cm}$, which corresponds to a spherical contraction volume of $4.2 \mathrm{~cm}^{3}$. Knowing the tissue volume and deposited energy before the start of contraction, we can estimate the energy density required for each cubic centimeter of treated tissue to reach tissue contraction effects. We can calculate that for $1 \mathrm{~L}$ of adipose tissue up to $48.3 \mathrm{~kJ}$ is required to start to see immediate and significant collagen contraction. These calculations of tissue energy needed to initiate adipose contraction are consistent with empirical data obtained with LAL treatment where energy from 50 up to $100 \mathrm{~kJ}$ is recommended for treatment of the abdominal area.

In vivo clinical monitoring of temperature in the adipose tissue and on the epidermal surface should allow the physician to predict more accurately the thermal treatment times and reduce the risk of thermal injuries.
Table 1 Average displacement and contraction threshold

\begin{tabular}{llll}
\hline & Dermis & Fascia & $\begin{array}{l}\text { Septa/Adipose } \\
\text { tissue }\end{array}$ \\
\hline Average displacement $(\mathrm{mm})$ & 2 & 2.75 & 6.5 \\
Threshold temperature $\left({ }^{\circ} \mathrm{C}\right)$ & 81.9 & 61.5 & 69.4 \\
$\begin{array}{l}\text { Time before start of contraction }(\mathrm{s}) \\
\text { Delivered energy before start of }\end{array}$ & 2.0 & 2.9 & 2.1 \\
\begin{tabular}{c} 
contraction $(\mathrm{J})$ \\
\hline
\end{tabular} & & 203 & 147 \\
\hline
\end{tabular}

\section{In Vivo Clinical RFAL Results}

The skin biopsies taken from an RFAL study patient at 12 months show normal dermal architecture with healthy collagen (Fig. 9) and elastin fibers (Fig. 10) in the deep reticular dermis and no evidence of scar tissue or abnormal collagen fibers. All RFAL patients demonstrated some level of contraction. From 8 to $15 \%$ linear tightening was observed at the end of the surgery on the operating table. It then increased dramatically during the first week when most of the swelling was reduced. The linear and area 


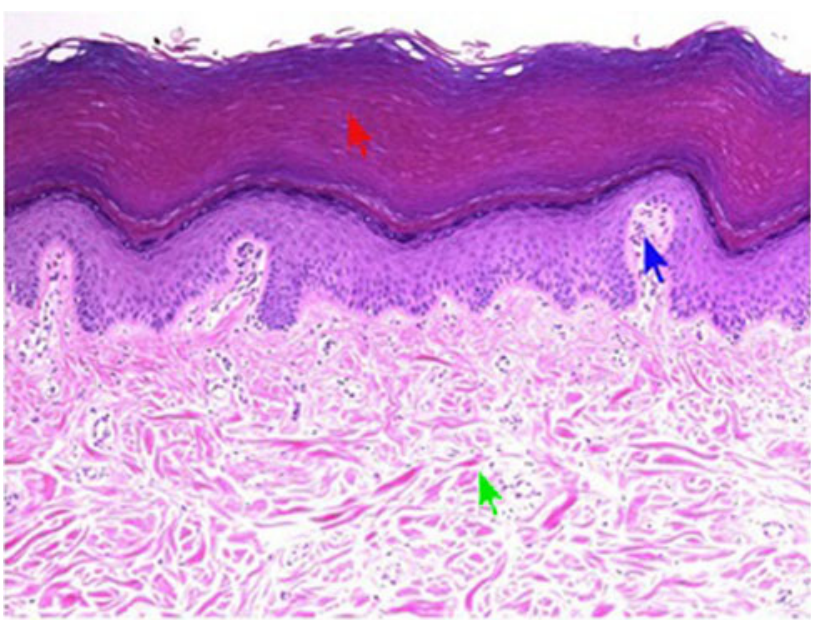

Stratum corneum is thick

\section{Papillary dermis}

\section{Reticular dermis}

Fig. 9 Normal skin histology 12 months following optimal RFAL thermal end point

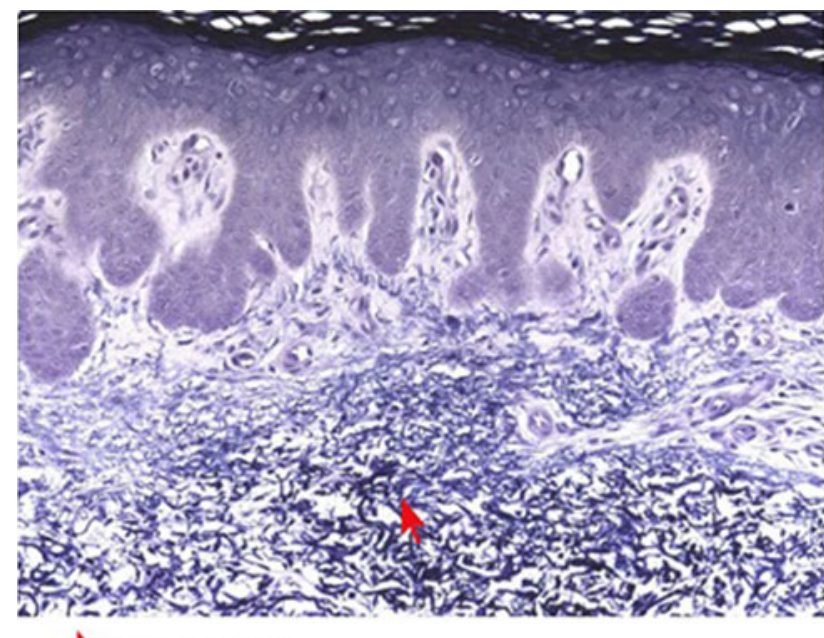

Elastic fibers

Fig. 10 Same RFAL patient with $43 \%$ contraction and normal elastic fiber content

contraction process continued for weeks and maximum contraction was noted at the last follow-up visit 24 weeks after the treatment.

Linear contraction observed at 6 months follow-up was much more significant than reported with any other technology and varied from 12.7 up to $47 \%$ depending on patient and treatment variables. It is important to note that soft tissue area contraction can be calculated as the square of the linear contraction and represents much higher numbers. The measured linear contraction was then correlated with three parameters: (1) aspirated volume that

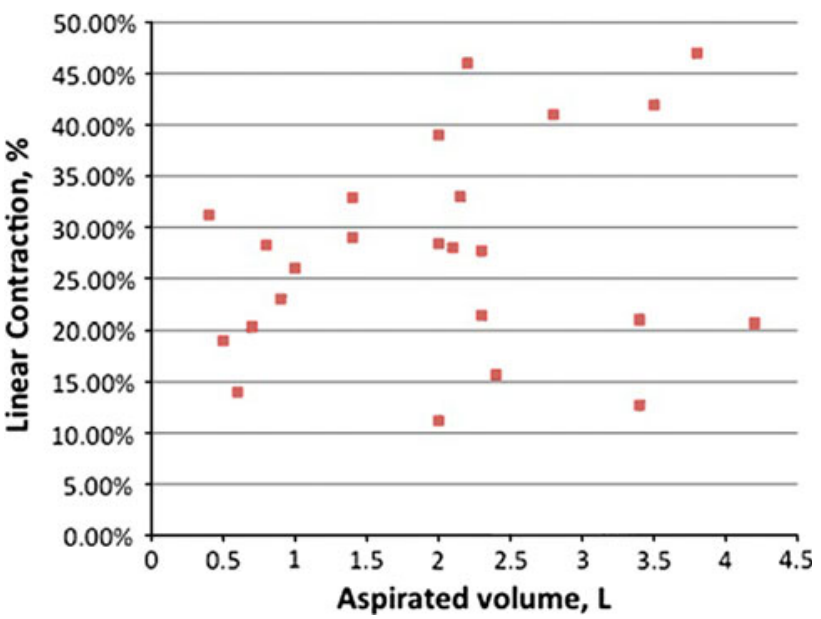

Fig. 11 Correlation between aspirated volume and linear contraction

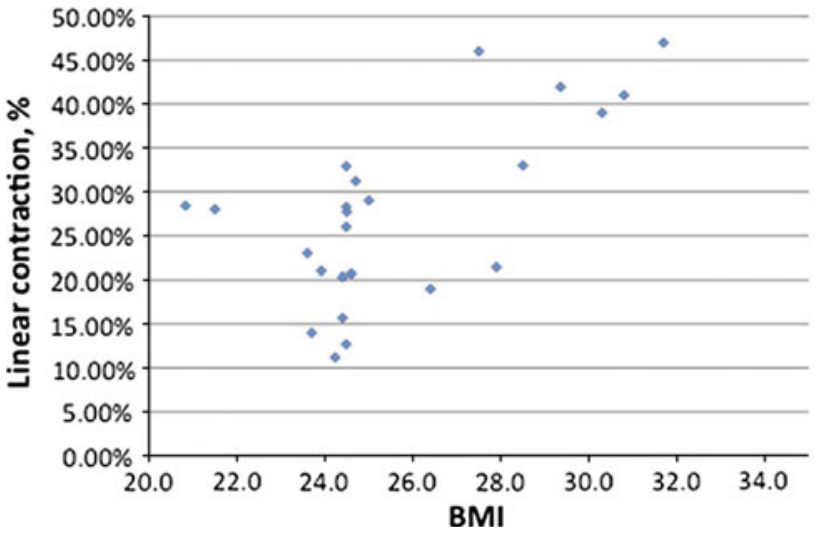

Fig. 12 Correlation between BMI and linear contraction

ranged from 0.5 to $3.4 \mathrm{~L}$, with an average volume of $2.0 \mathrm{~L}$, (2) BMI that varied from 20.8 to 31.7 , with an average index of 25.7, and (3) deposed RF energy that varied from 60 to $96 \mathrm{~kJ}$ per abdominal area, with an average RF energy of $72 \mathrm{~kJ}$.

For statistical analysis of the correlation between the measured variables and linear contraction, the Pearson product moment correlation coefficients were calculated. The closer the coefficient is to 1, the higher the linear correlation between the measured variable and tissue contraction. Analysis shows no or very weak correlation between aspirated volume and linear skin contraction. The Pearson coefficient is about 0.22. Figure 11 shows the correlation between these values and has a random distribution. The Pearson coefficient for correlation between contraction and patient BMI is much higher and equal to 0.64 . Figure 12 demonstrates a much stronger connection between these parameters and it is easy to understand that a patient with a larger volume of adipose tissue would have more tissue available to undergo contraction. 


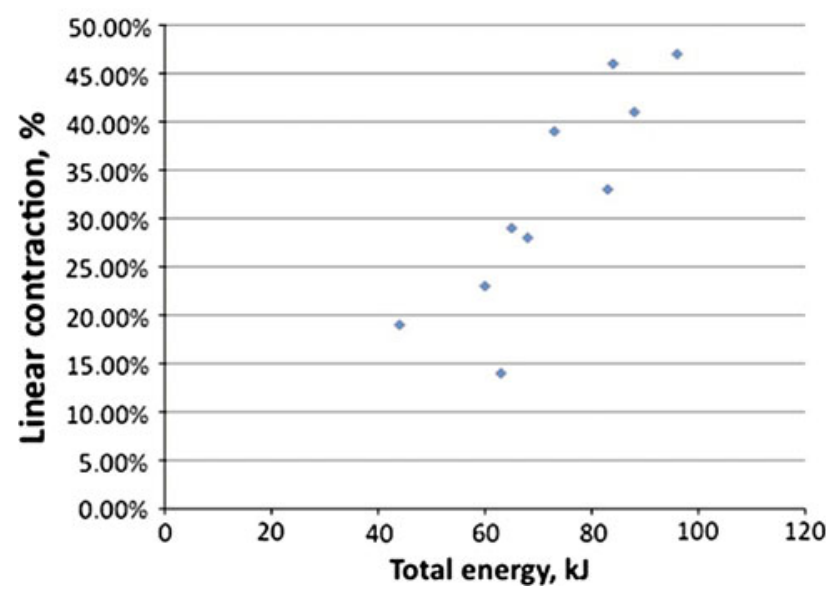

Fig. 13 Correlation between total energy and linear contraction

The highest correlation (0.86) was obtained between deposed RF energy and skin contraction. Figure 13 shows measurement results that have an almost linear function between these two parameters. The more energy deposited, the more linear contraction that was observed. In spite of improved contraction obtained at higher energies, the amount of energy used during treatment can and should be measured and controlled to avoid side effects such as seroma and skin burn and still achieve optimal linear and area contraction.

Features of an ideal liposuction procedure would include reduced ecchymosis, pain, and edema from preaspiration coagulation of adipose and vascular tissue, followed by less forceful and traumatic extraction forces, as well as significant soft tissue contraction when host tissue elasticity is compromised. Thermal-based lipoplasty appears to hold this potential.

In the present study based on volumetric heating, we reached an average local linear contraction of $31 \%$ that is statistically significantly higher than that reported with other energy-emitting liposuction technologies. Overall area contraction was much higher than linear contraction. We believe that these in vivo results confirm our proposed mechanism of RF-based tissue tightening and recruitment of the vertical and oblique fibrous adipose matrix. Our biopsy at 7 months suggests that the papillary and reticular dermis is populated with normal collagen and elastin that have been stimulated and remodeled by subnecrotic subdermal RFAL temperatures.

About $30 \%$ of patients noted minor weight loss but it is premature to correlate it with the treatment procedure.

The in vitro experiments produced different degrees of contraction for septal and dermal tissues which emphasizes the balance between these processes for optimal aesthetic results. Lower two-dimensional contraction of the skin and significant three-dimensional contraction of subdermal adipose connective tissue may cause wrinkling of the skin surface in high-volume liposuction patients.
During this study we had one case of a seroma that was treated with closed serial aspiration. Seroma is not a rare side effect for energy-assisted liposuction, especially for high-volume treatment and may necessitate a lower threshold for closed drainage systems in selected patients.

\section{Conclusions}

We believe the study results confirm the hypothesis of Kenkel [17], i.e., skin tightening and elasticity changes following thermal lipoplasty are mostly a result of subdermal tissue contraction but not dermal, which experiences lower heating during the treatment. It is clear that $40-42^{\circ} \mathrm{C}$ on the skin surface cannot result in an immediate contraction effect. Deep dermal remodeling may account for some horizontal contraction over time. It is possible that the dermal-fat junction experiences higher temperatures, but this process requires future investigation. We believe that the mechanism of subcutaneous collagen contraction during RF-assisted liposuction is similar to that witnessed in other types of collagen in that the contraction process has thermal contraction thresholds in the range of $60-70^{\circ} \mathrm{C}$.

It is likely more accurate to talk about tissue contraction rather than skin tightening because significant area contraction is a result of the strong contribution of deeper adipose fascial layers. Further studies with accurate 3D area measurements will tell us more about the RF-mediated area contraction in this RFAL technology. This RFAL thermal process and contraction can be effectively applied during a liposuction treatment in selected cases, improving patient satisfaction and extending liposuction procedures to higher-weight patients and patients with compromised skin conditions.

Disclosures Dr. Paul serves as consultant to and chairman of the board of the medical advisory board for Invasix, Ltd., and received consultation fees and stock options. He also serves as consultant to and chairman of the scientific advisory board for Angiotech/Surgical Specialties and receives consultant fees. Dr. Mulholland received consulting fees and technology from Invasix.

Open Access This article is distributed under the terms of the Creative Commons Attribution Noncommercial License which permits any noncommercial use, distribution, and reproduction in any medium, provided the original author(s) and source are credited.

\section{References}

1. Asbell PA, Maloney RK, Davidorf J, Hersh P, McDonald M, Manche E (2001) Conductive keratoplasty for the correction of hyperopia. Trans Am Ophthalmol Soc 99:79-87

2. Obrzut SL, Hecht P, Hayashi K, Fanton GS, Thabit G III, Markel MD (1998) The effect of radiofrequency on the length and 
temperature properties of the glenohumeral joint capsule. Arthroscopy 14(4):395-400

3. Lu Y, Edwards RB 3rd, Cole BJ, Markel MD (2001) Thermal chondroplasty with radiofrequency energy, an in vitro comparison of bipolar and monopolar radiofrequency devices. Am $\mathbf{J}$ Sports Med 29(1):42-49

4. Lu Y, Edwards RB 3rd, Kalscheur VL, Nho S, Cole BJ, Markel MD (2001) Effect of bipolar radiofrequency energy on human articular cartilage: comparison of confocal laser microscopy and light microscopy. Arthroscopy 17(2):117-123

5. Teruya TH, Ballard JL (2004) New approaches for the treatment of varicose veins. Surg Clin North Am 84:1397-1417

6. Doshi SN, Alster TS (2005) Combination radiofrequency and diode laser for treatment of facial rhytides and skin laxity. Cosmet Laser Ther 7:11-15

7. Emilia del Pino M, Rosado RH, Azuela A, Graciela Guzmán M, Argüelles D, Rodríguez C, Rosado GM (2006) Effect of controlled volumetric tissue heating with radiofrequency on cellulite and the subcutaneous tissue of the buttocks and thighs. J Drugs Dermatol 5(8):714-722

8. Fatemi A, Weiss MA, Weiss RA (2002) Short-term histologic effects of nonablative resurfacing: results with a dynamically cooled millisecond-domain $1320 \mathrm{~nm}$ Nd:YAG laser. Dermatol Surg 28(2):172-176
9. Mayoral FA (2007) Skin tightening with a combined unipolar and bipolar radiofrequency device. J Drugs Dermatol 6(2):212-215

10. Alster TS, Doshi SN, Hopping SB (2004) Combination surgical lifting with ablative laser skin resurfacing of facial skin: a retrospective analysis. Dermatol Surg 30(9):1191-1195

11. Zelickson B, Kist D, Bernstein E, Brown DB, Ksenzenko S, Burns J, Kilmer S, Mehregan D, Pope K (2004) Histological and ultrastructural evaluation of the effects of a radiofrequency-based nonablative dermal remodeling device: a pilot study. Arch Dermatol 140:204-209

12. Hsu T, Kaminer M (2003) The use of nonablative radiofrequency technology to tighten the lower face and neck. Semin Cutan Med Surg 22:115-123

13. DiBernardo B (2008) The best of hot topics-lipo-transfer and SmartLipo, ASAPS, May 6, 2008

14. Goldman A (2006) Submental Nd:YAG laser-assisted liposuction. Lasers Surg Med 38:181-184

15. Kim K, Geronemus R (2006) Laser lipolysis using a novel 1, 064 nm Nd:YAG laser. Dermatol Surg 32:241-248

16. Paul M, Mulholland RS (2009) A new approach for adipose tissue treatment and body contouring using radiofrequency-assisted liposuction. Aesth Plast Surg 33(5):687-694

17. Kenkel JM (2009) Evaluation of skin tightening after laser-assisted liposuction, commentary. Plast Reconstr Surg 29(5):407-408 